\title{
Wavelength-multiplexed memory based on a Lippmann architecture
}

\author{
K. Contreras, Z. Izri, C. Arnaud, G. Pauliat, G. Roosen \\ Laboratoire Charles Fabry de l'Institut d'Optique, CNRS, Univ Paris-Sud \\ Campus Polytechnique RD 128, Palaiseau Cedex, France.
}

It is well recognized that holographic data storage may represent the next generation of optical data storage. Unlike DVDs and other optical discs in which information is stored in a plane, holographic storage technologies have a volumetric memory. Among several configurations, the page-oriented architectures present great advantages, such as the high data rate that stems from their inherent parallelism [1]. In this work, we present such an architecture, based on the Lippmann photographic process [1-5], in which pages of data are stored with wavelength multiplexing.

In this scheme, during recording, the data-page is imaged onto a mirror set beneath the recording layer. Thus, the incident beam interferes with its reflection inside the thick recording medium and records a complex Bragg grating. After the mirror is removed, the plate is processed. Illuminating the plate with a plane wave at the same wavelength than the one used for recording allows reconstructing by Bragg-diffraction the whole page of data. Thanks to the wavelength selectivity of these Bragg gratings, several data-pages can be recorded in the same location using several wavelengths. The material thickness can be larger than the depth of focus of the images so that the light from the various pixels is allowed to interfere. This feature is the key to obtain large diffraction efficiencies and to get wavelength selective gratings. The recorded complex Bragg gratings thus present a wavelength selectivity similar to the one of a uniform Bragg grating of the same thickness so that the capacity of such a storage system is expected to be as large as those of conventional holographic storage devices [5].

In this communication, we will report on this storage capacity, and also on the first implementation of this architecture. The photograph of our current set-up is shown in Fig. 1a. An example of retrieved image is shown in Fig.1b.

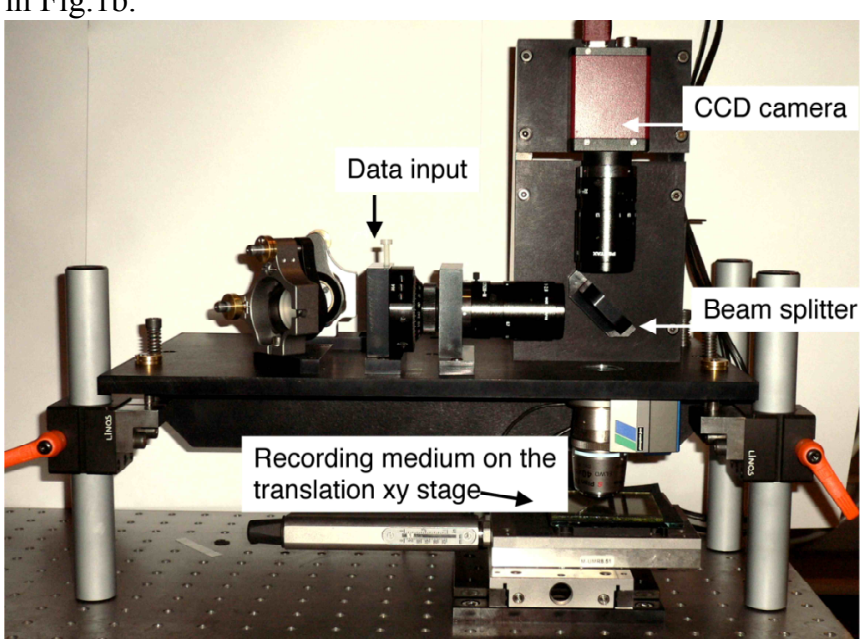

a)

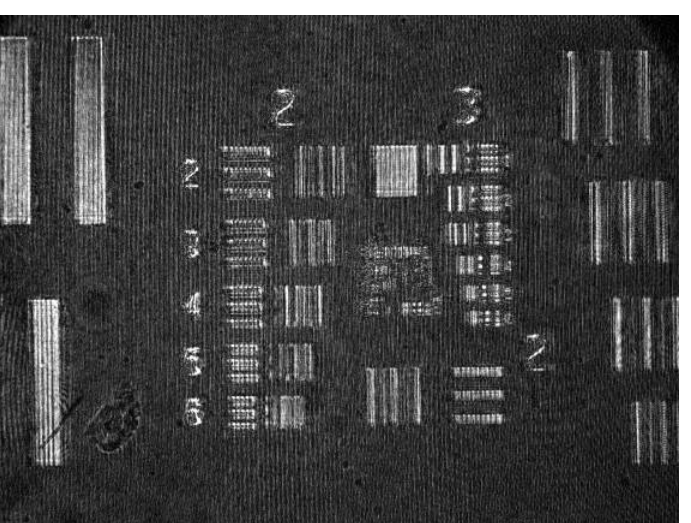

b)

Fig. 1: Photograph of the Lippmann architecture for wavelength multiplexing data-page oriented and b) retrieved image for one single wavelength. The total width of this image is equal to $480 \mu \mathrm{m}$.

\section{Acknowledgments}

K. Contreras gratefully acknowledges the support of the Programme Alban, the European Union Programme of High Level Scholarships for Latin America (E07D401978PE).

\section{References}

[1] H. J. Coufal, D. Psaltis, and G. T. Sincerbox, eds., Holographic Data Storage, Springer, Series in Optical Sciences (Springer-Verlag, 2000).

[2] G. Lippmann. "La photographie des couleurs". Comptes rendus de l'Académie des Sciences, T 112, pp. 274, Feb. 1891.

[3] H. Fleisher, P. Pengelly, J. Reynolds, R. Schools, and G. Sincerbox, "An optically accessed memory using the Lippmann process for information storage", Optical and Electro-Optical Information Processing (MIT Press, 1965).

[4] F. Guattari, G. Maire, K. Contreras, C. Arnaud, G. Pauliat, G. Roosen, S. Jradi, and C. Carré, "Balanced homodyne detection of Bragg microholograms in photopolymer for data storage," Opt. Express 15, 2234-2243 (2007)

[5] K. Contreras, G. Pauliat, C. Arnaud, G. Roosen, “Application of Lippmann interference photography to data storage”, J. Europ. Opt. Soc. Rap. Public. 08020 Vol. 3, 2008 